\title{
Forward to automatic forecasting and estimation of expected radiation hazards level
}

\section{Dorman Lev ${ }^{1}$}

Israel Cosmic Ray and Space Weather Center (ICRSWC), affiliated to Tel Aviv University, Shamir Research Institute, and Israel Space Agency, P.O. Box, 39040, Tel Aviv, ISRAEL 69978; and IZMIRAN, Moscow, Russia 108840; E-mail: lid010529@gmail.com

\section{Pustil'nik Lev}

ICRSWC, Tel Aviv, ISRAEL 69978; E-mail: levpust2149@gmail.com

Dai Uri

ICRSWC, Tel Aviv, ISRAEL 69978; E-mail: daiuri@gmail.com

\section{Keshtova Fatima}

Universitäten Oldenburg, GERMANY; E-mail: pantera@,realision.ru

\section{Sternlieb Abraham}

ICRSWC, Tel Aviv, ISRAEL 69978; E-mail: sarastar48@gmail.com

We present method of automatically forecasting of solar cosmic ray (SCR) impact caused by solar flare and estimation of radiation hazard level. We use model of solar cosmic ray diffusion in general form and coupling functions for neutron monitors network with different altitudes and cut-off rigidities, including also space detectors like GOES-11, 12. Another observational data for estimation of energy spectra is multiplicity of CR registration by some neutron monitors. We demonstrate algorithms for automatic estimation of the event starting, determination time-evolution of SCR in space by coupling functions in the frame of spectrographic method, solving inverse problem for SCR generation in solar corona and propagation in the interplanetary space, automatic determining on the basis of CR observation data parameters of SCR generation in solar corona, escaping into solar wind, and propagation in space. We show that on the basis of these parameters it can be automatic forecasting on the basis of first about 0.5 hour data expected level of radiation hazards for full time of event (up to about 48 hours) for objects in space on different distances from the Sun, in magnetosphere at different orbits, and in atmosphere at different altitudes and cutoff rigidities. If for some objects expected level of radiation hazards will be dangerous, after about 0.5 hour from event beginning will be formatted and sent corresponding Alert. We take into account that for the first about 0.5 hour data from event beginning, when coming mostly only small flux of high energy particles - radiation hazards expected very small in comparison with more delay time when coming main part of SCR with energy $\leq 1 \mathrm{GeV}$, formatted sufficient part of radiation hazards.

1

\section{Speaker}




\section{Introduction}

It is well known that in periods of great SEP (Solar Energetic Particle) events, the fluxes can be so big that memory of computers and other electronics in space may be destroyed, satellites and spaceships became dead (each year Insurance Companies paid billions dollars for these failures). Let us outlined that if it will be event as February 23, 1956 (see detail description in Dorman, 1957), will be destroyed about all satellites in few hours, the price of this will be more than 10-20 billion dollars, will be total destroying satellite communications and a rose a lot of other problems. In periods of great SEP events is necessary to switch off some part of electronics for short time to protect computer memories. These periods are also dangerous for astronauts on space-ships, and International Space Station (ISS), passengers and crew in commercial jets (especially during great radiation storms).

The problem is how to forecast exactly these dangerous phenomena. We show that exact forecast can be made by using high-energy particles (about 2-10 GeV/nucleon and higher) which transportation from the Sun is characterized by much bigger diffusion coefficient than for small and middle energy particles. Therefore high energy particles came from the Sun much more early (8-20 minutes after acceleration and escaping into solar wind) and later - main part of smaller energy particles caused dangerous situation for electronics and people health (about 60 and more minutes later).

As the first step, we use automatically working program "SEP-Start", supposed, developed and checked in the Emilio Segre' Observatory of Israel Cosmic Ray and Space Weather Center (Mt. Hermon, $2050 \mathrm{~m}$ above sea level, cut-off rigidity $10.8 \mathrm{GV}$ ). Using of this program on many CR stations and on satellites allowed determining automatically the beginning of SEP event.

The second step is "SEP-Coupling" - using developed in Dorman (1957, 1958, 2004) method of coupling functions for transformation obtained at different altitudes and cutoff rigidities data on CR intensity to the space and calculation CR energy spectrum and angle distribution out of the Earth's atmosphere and magnetosphere, directly in the interplanetary space near the Earth. Before we made these complicated operations step by step on the basis of historical SEP events data during long time and determined flare energetic particle spectrum in the interplanetary space and its change with time by method of coupling functions (in scientific literature called as Dorman functions). Now we prepared algorithms and try to create program which will be made these calculations automatically after each new minute of CR data very quickly for time not more than few seconds (Dorman et al., 206a).

The Third Step "SEP-Inverse Problem" is based on theoretically solved by Dorman et al. (2006b) about 10 years ago inverse problem and determine time of ejection energetic particles, source function and transport parameters in dependence from particle energy and distance from the Sun. Several years ago we made corresponding calculations very long time, so obtained results cannot be practically used for forecasting. Now we prepared all algorithms and try to create program which will be made these calculations automatically after each new minute of CR data very quickly for time not more than few seconds.

The Fourth Step "SEP-Forecasting" based on the theoretically solved direct problem and parameters founded in the Third Step and known coupling functions, we calculate time evolution of solar CR spectrum with time and expected total fluence (radiation hazards) in the interplanetary space for spaceships at different distances from the Sun in dependence of shielding, in the Earth's magnetosphere for satellites with different orbits, in the Earth's atmosphere for airplanes on different airlines in dependence of altitude and cutoff rigidities, and for ground at different air pressure and cutoff rigidities. Again, we checked all these mathematical procedures basing on real data of historical SEP events and it need so long time that it was not possible to use these results for forecasting of expected radiation hazards. Now we for this step also prepared all algorithms and try to create program which will be made these calculations automatically after each new minute of CR data very quickly for time not more than few seconds. To determine the quality of obtained results, after 5-10 minutes from beginning starts to work the final, Fives Step.

The Fives Step "Checking of Forecasting Quality and Alerts" starts to work at 5-10 minutes after the event beginning. In this Step we compare expected (calculated in the Fourth Step by using coupling functions CR intensity for neutron monitors on different stations and on satellites) with observed. If the difference will be small enough (smaller than 10-20\%) and the radiation hazards expected to be dangerous for spaceships in the interplanetary space, for some satellites in the Earth's magnetosphere, for airplanes on some airlines or for some objects on the ground, will be send corresponding Alerts with detail information on the expected radiation hazards calculated in the Fourth Step. After few minutes a 
new, more exact Alerts will be sent. More and more exact new Alerts will be repeated each few minutes during the dangerous event. Let us outline that Step 1 finished to work after determining the beginning of SEP event, but Steps $2-5$ continued to work for each new minute. They will be finished to work only when the difference between consequences Alerts became much smaller than statistical errors.

\section{What we need to prepare before start the automatic forecasting procedure?}

2.1. Data what we need to use for automatic on-line forecasting procedure. We will use one-minute CR data, available from Internet (first of all - NMDB data): high-latitude stations with $R_{C}<1 \mathrm{GV}$ (Oulu, Apatite, South Pole, and some other), middle-latitude stations with $R_{C}=2-4 \mathrm{GV}$ (Moscow, Kiel, Novosibirsk, Yakutsk, Lomnitsky Stit, Yungfraujokh, and others), stations with $R_{C}=6-7 \mathrm{GV}$ (Rome, Athens, Tjan Shan, and others), and low-latitude stations with $R_{C}=10-16$ GV (Mt. Hermon, Mt. Norikura, Mexico, Haleacala, and others). For very small energy solar CR we will use satellite one-minute data, also available from Internet (e.g., GOES data). For automatically worked forecasting system we need to prepare also following:

2.2. For each used CR stations we need to know exactly values of cutoff rigidities $R_{c}$ and how they change with secular variations of the main geomagnetic field and with magnetic activity. Now in IZMIRAN are calculated planetary distribution of $R_{c}$ during the period $1950-2050$ in dependence of the level of magnetic activity (Gvozdevsky et al., 2017).

2.3. For each used CR stations we need to know exact values of atmospheric pressure $\boldsymbol{h}$ (in units $1000 \mathrm{~g} / \mathrm{cm}^{2}$ ) at the point of CR detector.

2.4. For each used CR stations we need to calculate coupling functions according to following formulas. As it was shown in many CR expeditions and numerical calculations of meson-nuclear cascades in the Earth's atmosphere (Dorman, 2004, 2009), for the polar normalized coupling function (at $R_{c}=0$ ) for any secondary component of type $i$ ( $i=n-$ for total neutron component, $i=m-$ for neutron multiplicities $m=1,2,3, \ldots, i=h \mu$ - for hard muons, $i=s \mu$-for soft muons, $i=e p-$ for electron-photon component, and so on) can be approximated by the special function

$$
W_{o i}(R, h)=a_{i}(h) k_{i}(h) R^{-\left(k_{i}(h)+1\right)} \exp \left(-a_{i}(h) R^{-k_{i}(h)}\right),
$$

and for the normalized coupling functions at any point on Earth with cut off rigidity $R_{c}$ will be

$$
W_{i}\left(R_{c}, R, h\right)= \begin{cases}0 & \text { if } R<R_{c} \\ a_{i}(h) k_{i}(h) R^{-\left(k_{i}(h)+1\right)}\left(1-a_{i}(h) R_{c}^{-k_{i}(h)}\right)^{-1} \exp \left(-a_{i}(h) R^{\left.-k_{i}(h)\right)} \text { if } \quad R \geq R_{c} .\right.\end{cases}
$$

Coupling function connects observed relative CR variation $\Delta N_{i}\left(h, R_{c}, t\right) / N_{i 0}\left(h, R_{c}, t_{0}\right)$ by any detector in the Earth's atmosphere at pressure $\mathrm{h}$ with primary $\mathrm{CR}$ variation in space relative to primary galactic CR spectrum $\Delta D(R, t) / D_{G}\left(R, t_{0}\right)$. Here $N_{i 0}\left(h, R_{c}, t_{0}\right)$ is measured counting rate before the SEP event, and $\Delta N_{i}\left(h, R_{c}, t\right)=N_{i}\left(h, R_{c}, t\right)-N_{i 0}\left(h, R_{c}, t_{0}\right)$, i.e. the change of detector's counting rate during the SEP event. The primary galactic CR spectrum before SEP event is $D_{G}\left(R, t_{0}\right)$, and the primary CR variation in space is $\Delta D(R, t)=D(R, t)-D_{G}\left(R, t_{0}\right)$. In this case according to method of coupling functions (Dorman, 1957, 2004) observed relative CR variation will be

$$
\Delta N_{i}\left(h, R_{c}, t\right) / N_{i 0}\left(h, R_{c}, t_{0}\right)=\int_{R_{c}}^{\infty} W_{i}\left(R_{c}, R, h\right)\left[\Delta D(R, t) / D_{G}\left(R, t_{0}\right)\right] d R
$$

The dependence of $a_{n}$ and $k_{n}$ for total neutron component and $a_{m}$ and $k_{m}$ for neutron multiplicities $m=1,2,3, \ldots$ on the CR station with pressure $h$ (in atm $=1000 \mathrm{~g} / \mathrm{cm}^{2}$ ) and solar activity level 
characterized by the logarithm of CR intensity per one hour (we used here monthly averaged of Climax $\mathrm{NM} \ln \left(N_{\mathrm{Cl}}\right)$, available starting from 1952) can be approximated by the functions (Dorman, 2004):

$$
\begin{gathered}
a_{n}=\left(-2.915 h^{2}-2.237 h-8.654\right) \ln \left(N_{\mathrm{Cl}}\right)+\left(24.584 h^{2}+19.460 h+81.230\right), \\
k_{n}=\left(0.180 h^{2}-0.849 h+0.750\right) \ln \left(N_{\mathrm{Cl}}\right)+\left(-1.440 h^{2}+6.403 h-3.698\right),
\end{gathered}
$$

$$
a_{m}=\left[\left(-2.915 h^{2}-2.237 h-8.638\right) \ln \left(N_{\mathrm{Cl}}\right)+\left(24.584 h^{2}+19.46 h+81.23\right)\right) \times\left(0.987 m^{2}+0.225 m+6.913\right) / 9.781 \text {, }
$$

$$
k_{m}=\left[\left(0.180 h^{2}-0.849 h+0.750\right) \ln \left(N_{\mathrm{Cl}}\right)+\left(-1.440 h^{2}+6.403 h-3.698\right)\right] \times(0.081 m+1.819) / 1.940
$$

where multiplicities $m=1,2,3, \ldots$ Instead of Climax NM, one can also use monthly averages of any other CR station with appropriate recalculation of the coefficients determined by correlation between monthly data $N_{\mathrm{Cl}}$ of Climax NM and this station for several years. For example, the recalculated coefficients for ESOI $6 \mathrm{NM}-64$ are

$$
\ln \left(N_{\mathrm{Cl}}\right)=(1.947 \pm 0.022) \times \ln \left(N_{\mathrm{ESOI}}\right)-(1.75 \pm 0.11) ; C C=0.892 \pm 0.003 .
$$

For Rome 17NM-64 these recalculation coefficients are

$$
\ln \left(N_{\mathrm{Cl}}\right)=(2.048 \pm 0.005) \times \ln \left(N_{\text {Roma }}\right)-(1.965 \pm 0.024) ; C C=0.9819 \pm 0.0004 \text {. }
$$

For 18 NM-64 Apatite the found recalculation coefficients are

$$
\ln \left(N_{\mathrm{Cl}}\right)=(1.178 \pm 0.003) \times \ln \left(N_{\text {Apat }}\right)-(2.256 \pm 0.016) ; C C=0.9922 \pm 0.0002 \text {. }
$$

For Athens station with $6 \mathrm{NM}-64$ the recalculation coefficients are

$$
\ln \left(N_{\mathrm{Cl}}\right)=(2.278 \pm 0.017) \times \ln \left(N_{\text {Ath }}\right)-(0.828 \pm 0.069) ; C C=0.947 \pm 0.002 \text {. }
$$

For Moscow station with 24 NM-64 the recalculation coefficients are

$$
\ln \left(N_{\mathrm{Cl}}\right)=(1.194 \pm 0.003) \times \ln \left(N_{\mathrm{Mosc}}\right)+(1.853 \pm 0.017) ; C C=0.9845 \pm 0.0003 \text {. }
$$

For McMurdo station with $18 \mathrm{NM}-64$ the recalculation coefficients are

$$
\ln \left(N_{\mathrm{Cl}}\right)=(1.121 \pm 0.002) \times \ln \left(N_{\text {McMur }}\right)+(2.074 \pm 0.010) ; C C=0.9805 \pm 0.0002 \text {. }
$$

For Kiel station with 18 NM-64 the recalculation coefficients are

$$
\ln \left(N_{\mathrm{Cl}}\right)=(1.152 \pm 0.002) \times \ln \left(N_{\mathrm{Kiel}}\right)+(2.395 \pm 0.010) ; C C=0.9764 \pm 0.0002 \text {. }
$$

For Oulu station with 18 NM-64 the recalculation coefficients are

$$
\ln \left(N_{\mathrm{Cl}}\right)=(1.1794 \pm 0.0013) \times \ln \left(N_{\text {Oulu }}\right)+(2.8424 \pm 0.0013) ; C C=0.9915 \pm 0.0001 \text {. }
$$

For Almata (high-mountain) station with 24 NM-64 the recalculation coefficients are

$$
\ln \left(N_{\mathrm{Cl}}\right)=(1.875 \pm 0.004) \times \ln \left(N_{\text {Almata }}\right)-(5.277 \pm 0.029) ; C C=0.9746 \pm 0.0003 \text {. }
$$

Substituting Eqs. 2.7 - 2.15 in Eqs. 2.3-2.6, we obtain the coupling functions as a function of the level of solar activity on the basis of monthly NM data of ESOI, Rome, Athens, Moscow, McMurdo, Kiel, Oulu, Almata. By this way it is easy to find corresponding recalculation coefficients for any CR station with NM in the World.

Because in our method of determining spectrum of solar CR out of magnetosphere can be used also other CR secondary components, let us outlined that according to Dorman (2004), the coefficients $a_{i}, k_{i}$ in the analytical form described by Eq. 2.1 for different CR secondary components will be as 
following: 1) for neutron component at $h_{o}=312 \mathrm{mb}$ (about $10 \mathrm{~km}$ - altitude of many airlines) $a_{n}=8.30, k_{n}=1.45 ; 2$ ) for neutron component at $h_{o}=680 \mathrm{mb}$ (mountains at about $3 \mathrm{~km}$ ), $a_{n}=13.62, k_{n}=1.26$; 3) for hard muon component at sea level $h_{o}=1030 \mathrm{mb}$ $a_{h \mu}=35.3, k_{h \mu}=0.95$; and 4) for hard muon component underground at the depth $7 \mathrm{~m}$ w.e., $a_{h \mu}=58.5, k_{h \mu}=0.94$.

4.5. We need tabulate special functions $F_{i}\left(R_{c}, \gamma\right)$ for each used CR station. Let us suppose that in some interval of rigidities to which are sensitive our detectors the primary solar CR variation $\Delta D(R, t)$ we describe as power function

$$
\Delta D(R, t) / D_{G}(R)=b(t) R^{-\gamma(t)},
$$

where $D_{G}(R)$ is the differential spectrum of galactic CR at $t=0$ (for which coupling functions are defined). This form of spectrum was observed at high energy region (more than $\sim 1 \mathrm{GeV}$ ) practically in about all SEP events (Dorman, 1957, 1978; Dorman and Miroshnichenko, 1968; Miroshnichenko, 2015). In Eq. 2.16 the parameters $b(t), \gamma(t)$ depend on $t$ and we will try to determine them from CR ground and satellite observations. Let us put Eq. 2.1b and Eq. 2.16 into Eq. 2.2. For calculating on-line expected spectrum of solar CR out of the Earth atmosphere and magnetosphere we need to know for each used CR station and each secondary component functions

$$
\Delta N_{i}\left(h, R_{c}, t\right) / N_{i 0}\left(h, R_{c}, t_{0}\right)=b(t) F_{i}\left(R_{c}, \gamma\right)=b(t) a_{i} k_{i}\left(1-\exp \left(-a_{i} R_{c}^{-k_{i}}\right)\right)^{-1} \int_{R_{c}}^{\infty} R^{-\left(k_{i}+1+\gamma\right)} \exp \left(-a_{i} R^{-k_{i}}\right) d R
$$

where $R_{c}, a_{i}, k_{i}$ were described above (for NM by Eqs. $2.3-2.6$ ). Function $F_{i}\left(R_{c}, \gamma\right)$ can be calculated by replacing variables through not full gamma function:

$$
F_{i}\left(R_{c}, \gamma\right)=a_{i}^{-\gamma / k_{i}} \gamma\left(\frac{\gamma}{k_{i}+1} ; a_{i} R_{c}^{-k_{i}}\right)\left(1-\exp \left(-a_{i} R_{c}^{-k_{i}}\right)\right)^{-1} .
$$

2.6. For using on-line data of pairs CR stations with different cutoff rigidities we need to tabulate special functions $\Psi_{k l}\left(R_{c 1}, R_{c 2}, \gamma\right)$. In this case the ratio of CR enhancements on both stations will be

Where

$$
\delta N_{k}\left(R_{c 1}, t\right) / \delta N_{l}\left(R_{c 2}, t\right)=\Psi_{k l}\left(R_{c 1}, R_{c 2}, \gamma\right)
$$

$$
\begin{aligned}
& \Psi_{k l}\left(R_{c 1}, R_{c 2}, \gamma\right)=F_{k}\left(R_{c 1}, \gamma\right) / F_{l}\left(R_{c 2}, \gamma\right) \\
& \quad=a_{k}{ }^{-\gamma / k} k \gamma\left(\frac{\gamma}{k_{k}+1} ; a_{k} R_{c 1}^{-k_{k}}\right)\left(1-\exp \left(-a_{k} R_{c 1}^{-k_{k}}\right)\right)^{-1} / a_{l}^{-\gamma / k} l \gamma\left(\frac{\gamma}{k_{l}+1} ; a_{l} R_{c 2}{ }^{k} l\right)\left(1-\exp \left(-a_{l} R_{c 2}^{-k_{l}}\right)\right)^{-1} .
\end{aligned}
$$

After tabulating $\Psi_{k l}\left(R_{c 1}, R_{c 2}, \gamma\right)$ according to Eq. 2.20, we calculate the corresponding inverse function on the basis of Eq. 2.19:

$$
\gamma(t)=\Omega_{k l}\left(\delta N_{k}\left(R_{c 1}, t\right) / \delta N_{l}\left(R_{c 2}, t\right)\right) \text {. }
$$

As some examples, on Figure 1 are shown Function (2.21) for ratio of CR intensity increasing on stations Tjan Shan to Moscow. 
Fig. 1. An example of determining $\gamma(t)$ (ordinate axes) by ratio $\delta N_{k}\left(R_{c 1}, t\right) / \delta N_{l}\left(R_{c 2}, t\right)$ for stations Tjan Shan to Moscow (abscissa axes. From Fig. 1 follows that $\gamma(t)=2.389 \ln$ (Tjan Shan/Moscow $)+0.842 ; \mathrm{CC}^{2}=0.9922$.

After determining $\gamma(t)$ according to Eq. 2.21, functions $b(t)$ and $\Delta D(R, t)$ can be determined from:

$$
\begin{gathered}
b(t)=\delta N_{m}\left(R_{c 1}, t\right) / F_{m}\left(R_{c 1}, \gamma(t)\right)=\delta N_{n}\left(R_{c 2}, t\right) / F_{n}\left(R_{c 2}, \gamma(t)\right) . \\
\Delta D(R, t)=b(t) D_{G}(R) R^{-\gamma(t)}
\end{gathered}
$$

\section{Algorithms for the first stage: automatically determining the start of SEP event}

The determination of increasing flux we suppose to make by comparison with intensity averaged for one day (1440 minutes) from 1560 to 120 minutes before the present $Z$-th one-minute data. For each $\mathrm{Z}$ minute data, start the program "SEP-Search". The program for each $Z$-th minute determines the values

$$
\begin{gathered}
D_{A Z}=\left[\ln \left(I_{A Z}\right)-\sum_{k=Z-1560}^{k=Z-120} \ln \left(I_{A k}\right) / 1440\right] / \sigma, \\
D_{B Z}=\left[\ln \left(I_{B Z}\right)-\sum_{k=Z-1560}^{k=Z-120} \ln \left(I_{B k}\right) / 1440\right] / \sigma,
\end{gathered}
$$

where $I_{A k}$ and $I_{B k}$ are one-minute counting rate in the sections of neutron super- monitor $A$ and $B$, and $\sigma$ is the statistical error for one minute:

$$
\sigma=\left\langle I_{A k}\right\rangle^{-1 / 2} \cong\left\langle I_{B k}\right\rangle^{-1 / 2}
$$

For NM at Mt. Hermon the average counting rate in each sections $\left\langle I_{A k}\right\rangle \cong\left\langle I_{B k}\right\rangle$ is about 5000 per one minute, and $\sigma \cong 0.014=1.4 \%$. If simultaneously

$$
D_{A Z} \geq 2.5, D_{B Z} \geq 2.5,
$$

the program "SEP-Search" repeat the calculation for the next Z+1-th minute and if Eq. 3.4 is satisfied again, the onset of great SEP is determined and starts the program "SEP-Research/Spectrum". In this case we use the last value of average intensities in both sections, determined on the basis of one day (1440 minute data). If Eq. 3.4 is not satisfied, the program "SEP-Search" continue to check next minutes up to the moment when Eq. 3.4 will be satisfied at least for two nearest minutes.

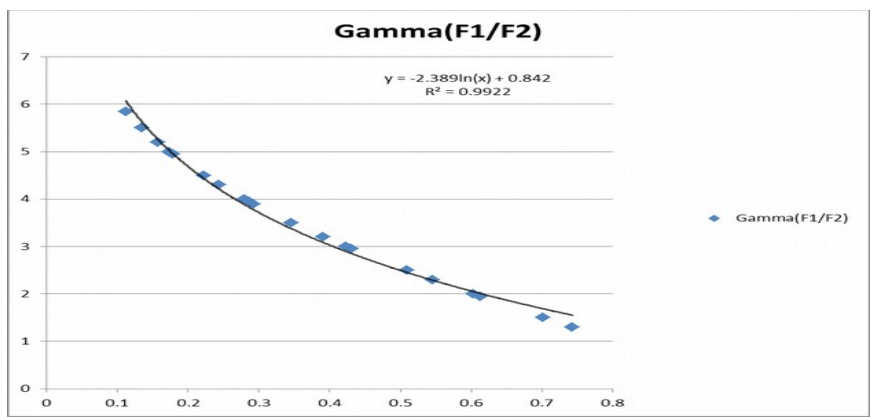

4. The inverse problem for the case when diffusion coefficient depends from particle rigidity and from the distance to the Sun. When we analyzed data of the SEP event at 29 September 1989, we obtained the strange big increasing of diffusion coefficient with time during this event. From obtained information we came to conclusion that it does not mean real increasing diffusion coefficient for so short time, but it means increasing diffusion coefficient with increasing the distance from the Sun with propagation SEP in the interplanetary space. Let us suppose, according to Parker (1965), that the diffusion coefficient

$$
\kappa(R, r)=\kappa_{1}(R) \times\left(r / r_{1}\right)^{\beta} .
$$

In this case the solution of diffusion equation will be according to Parker (1965) 


$$
N(R, r, t)=\frac{N_{o}(R) \times r_{1}^{3 \beta /(2-\beta)}\left(\kappa_{1}(R) t\right)^{-3 /(2-\beta)}}{(2-\beta)^{(4+\beta) /(2-\beta)} \Gamma(3 /(2-\beta))} \times \exp \left(-\frac{r_{1}^{\beta} r^{2-\beta}}{(2-\beta)^{2} \kappa_{1}(R) t}\right),
$$

where $t$ is the time after SEP ejection into solar wind. So now we have four unknown parameters: time of SEP ejection into solar wind $T_{e}, \beta, \kappa_{1}(R)$, and $N_{o}(R)$. Let us assume that according to ground and satellite measurements at the distance $r=r_{1}=1$ AU from the Sun we know $N_{1}(R), \quad N_{2}(R), \quad N_{3}(R), \quad N_{4}(R)$ at UT times $T_{1}, T_{2}, T_{3}, T_{4}$. In this case

$$
t_{1}=T_{1}-T_{e}=x, t_{2}=T_{2}-T_{1}+x, t_{3}=T_{3}-T_{1}+x, t_{4}=T_{4}-T_{1}+x,
$$

For each $N_{i}\left(R, r=r_{1}, T_{i}\right)$ we obtain from Eq. 4.2 and Eq. 4.3:

$$
N_{i}\left(R, r=r_{1}, T_{i}\right)=\frac{N_{o}(R) \times r_{1}^{3 \beta /(2-\beta)}\left(\kappa_{1}(R)\left(T_{i}-T_{1}+x\right)\right)^{-3 /(2-\beta)}}{(2-\beta)^{(4+\beta) /(2-\beta)} \Gamma(3 /(2-\beta))} \times \exp \left(-\frac{r_{1}^{2}(2-\beta)^{-2}}{\kappa_{1}(R)\left(T_{i}-T_{1}+x\right)}\right) \text {, }
$$

where $i=1,2,3$, and 4 . To determine $x$ let us step by step exclude unknown parameters $N_{o}(R)$, $\kappa_{1}(R)$, and then $\beta$. As result, we obtain equation for determining $x$ and then by Eq. 4.3 determine times $t_{1}, t_{2}, t_{3}$, and $t_{4}$ of data relative to SEP injection into solar wind. After this we determine:

$$
\beta=2-3\left[\left(\ln \left(t_{2} / t_{1}\right)\right)-\frac{t_{3}\left(t_{2}-t_{1}\right)}{t_{2}\left(t_{3}-t_{1}\right)} \ln \left(t_{3} / t_{1}\right)\right] \times\left[\left(\ln \left(N_{1} / N_{2}\right)\right)-\frac{t_{3}\left(t_{2}-t_{1}\right)}{t_{2}\left(t_{3}-t_{1}\right)} \ln \left(N_{1} / N_{3}\right)\right]^{-1} .
$$

Then we determine unknown parameter $\kappa_{1}(R)$ in Eq. 4.1:

$$
\kappa_{1}(R)=\frac{r_{1}^{2}\left(t_{1}^{-1}-t_{2}^{-1}\right)}{3(2-\beta) \ln \left(t_{2} / t_{1}\right)-(2-\beta)^{2} \ln \left(N_{1} / N_{2}\right)}=\frac{r_{1}^{2}\left(t_{1}^{-1}-t_{3}^{-1}\right)}{3(2-\beta) \ln \left(t_{3} / t_{1}\right)-(2-\beta)^{2} \ln \left(N_{1} / N_{3}\right)} .
$$

After determining parameters $\beta$ and $\kappa_{1}(R)$ we can determine the last unknown parameter, source function $N_{o}(R)$ :

$$
N_{o}(R)=N_{i}(2-\beta)^{(4+\beta) /(2-\beta)} \Gamma(3 /(2-\beta)) r_{1}^{-3 \beta /(2-\beta)}\left(\kappa_{1}(R) t_{i}\right)^{3 /(2-\beta)} \times \exp \left(\frac{r_{1}^{2}}{(2-\beta)^{2} \kappa_{1}(R) t_{i}}\right) \text {, }
$$

where index $i=1,2$ or 3 . Let us outline that for all $i$ results must be the same (it can be used as control of correct calculations.

\section{Algorithms for Comparison with Observations and Estimation of Expected Radiation Hazards}

To check our forecasting we compare obtained results in the Stage 3 with observations at the beginning of $\mathrm{SEP}$ event. Expected variation of $\mathrm{CR}$ intensity will be for $\mathrm{NM}$ on the ground

$$
N_{i}\left(R_{c}, h, t\right)=\int_{R_{c}(t)}^{\infty} m_{i}(R, h) \frac{N_{o}(R) \times r_{1}^{3 \beta /(2-\beta)}\left(\kappa_{1}(R)\left(T_{i}-T_{1}+x\right)\right)^{-3 /(2-\beta)}}{(2-\beta)^{(4+\beta) /(2-\beta)} \Gamma(3 /(2-\beta))} \times \exp \left(-\frac{r_{1}^{2}(2-\beta)^{-2}}{\kappa_{1}(R)\left(T_{i}-T_{1}+x\right)}\right) d R
$$

Comparison with neutron monitor and satellite data (see Figures 2 and 3) shows that using about 20-30 first minutes of SEP event gives good agreement with all observed data up to about 48 hours. 

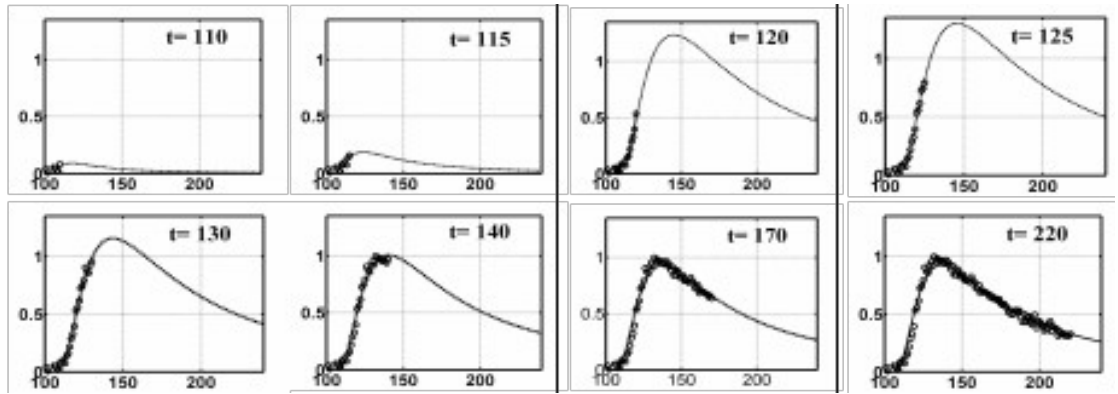

Fig. 2. Calculation on line parameters $\beta, \kappa_{1}(R)$, and $N_{o}(R)$ and forecasting of total neutron intensity (on the abscissa axis the time $t$ is in minutes after 10.00 UT of September 29, 1989). Curves -forecasting on the basis of the first 10 minutes, then on the basis of the first 15 minutes, then on the basis of the first 20 minutes, then on the basis of the first 25 minutes, then on the basis of the first 30 minutes, and so on. Small circles - observations one minute data.

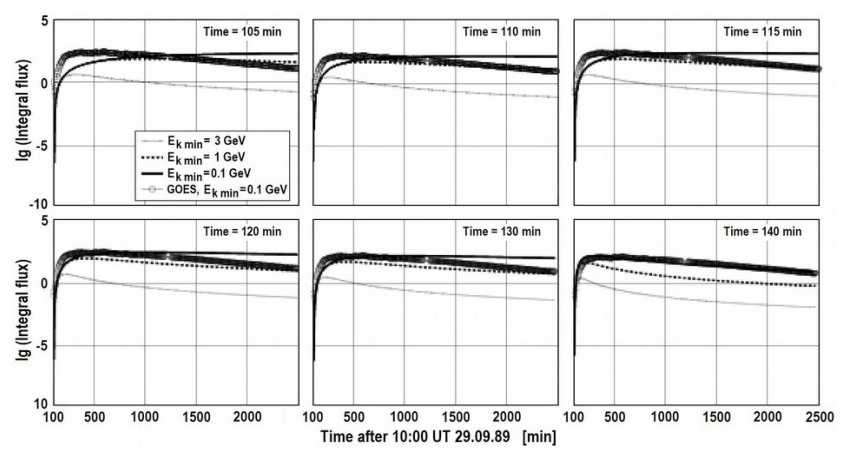

Fig. 3. Combined forecasting on the basis of NM data and beginning of satellite data; comparison with GOES observations. Curves forecasting of solar CR intensity with kinetic energy $>3 \mathrm{GeV},>1 \mathrm{GeV}$, and $>0.1 \mathrm{GeV}$. Circles - observations on GOES of SEP intensity with energy $>0.1 \mathrm{GeV}$. It can be seen that after 5 minutes it is a big difference between forecasting and observed variations of solar $\mathrm{CR}$ intensity with kinetic energy $>0.1 \mathrm{GeV}$ (in this short time diffusion regime does not formatted), but with time the coincidence became more close and fully coincident after 40 minutes up to about two days from the beginning of SEP event.

Forecasting of expected radiation hazards for space-crafts in the interplanetary space. In this case the $R_{c}=0$ and $R_{\min }$ will be determined only by shielding $R_{s}=R_{\min }$. The full radiation hazard during SEP event for distance $r$ from the Sun will be determined by following fluency:

$$
F(r)=\int_{0}^{\infty} d t \int_{R_{\min }}^{\infty} \frac{N_{o}(R) \times r^{3 \beta /(2-\beta)}\left(\kappa_{1}(R) t\right)^{-3 /(2-\beta)}}{(2-\beta)^{(4+\beta) /(2-\beta)} \Gamma(3 /(2-\beta))} \times \exp \left(-\frac{r^{2}(2-\beta)^{-2}}{\kappa_{1}(R) t}\right) d R .
$$

Forecasting of expected radiation hazards for satellites in the Geomagnetosphere. In this case the $R_{c}$ $\neq 0$ and $R_{\min }=\max \left(R_{c}, R_{s}\right)$. The full radiation hazard during SEP event will be determined by following fluency:

$$
F\left(r_{1}\right)=\int_{0}^{\infty} d t \int_{R_{\min }(t)}^{\infty} N_{o}(R) \times \frac{r_{1}^{3 \beta /(2-\beta)}\left(\kappa_{1}(R) t\right)^{-3 /(2-\beta)}}{(2-\beta)^{(4+\beta) /(2-\beta)} \Gamma(3 /(2-\beta))} \times \exp \left(-\frac{r_{1}^{2}(2-\beta)^{-2}}{\kappa_{1}(R) t}\right) d R
$$

Forecasting of expected radiation hazards for air-crafts in troposphere and stratosphere as well as on the ground. In this case the $R_{c} \neq 0$ and $R_{\min }=\max \left(R_{c}, R_{s}\right)$. The full radiation hazard during SEP event will be determined by following fluency:

$$
F\left(h, r_{1}\right)=\int_{0}^{\infty} d t \int_{R_{\min }(t)}^{\infty} m(R, h) N_{o}(R) \times \frac{r_{1}^{3 \beta /(2-\beta)}\left(\kappa_{1}(R) t\right)^{-3 /(2-\beta)}}{(2-\beta)^{(4+\beta) /(2-\beta)} \Gamma(3 /(2-\beta))} \times \exp \left(-\frac{r_{1}^{2}(2-\beta)^{-2}}{\kappa_{1}(R) t}\right) d R
$$

Automatically formatting corresponding Alerts when Radiation Hazards expected to be dangerous for electronics or/and people health

In all cases, when the calculated radiation hazards expected to be dangerous for electronics, computer memories, or/and for people health, will be immediately send automatically formatted corresponding Alerts with information on the calculated in Section 8 expected fluencies for different cases. 
Acknowledgements. We use this opportunity to acknowledge Ministry of Science of State Israel, Tel Aviv University, Israel Space Agency, and Shamir Institute for Research for continues support of Israeli Cosmic Ray \& Space Weather Centre.

\section{References.}

Dorman L.I., Cosmic Ray Variations. Gostekhteorizdat, Moscow , pp 495. In Russian. 1957.

In English: US Department of Defense, Washington, DC. 1958.

Dorman L.I., Miroshnichenko L.I., "Solar Cosmic Rays”, Physmat Press, Moscow (in Russian), 1968.

Dorman L.I., Cosmic Rays of Solar Origin, Moscow, 1978.

Dorman L.I., Zukerman I., "Initial concept for forecasting the flux and energy spectrum of energetic particles using ground-level cosmic ray observations", Advances in Space Research, Volume 31, Issue 4, p. 925-932, 2003,

Dorman L.I., Cosmic Rays in the Earth's Atmosphere and Underground. Kluwer Ac. Publ., Dordrecht. 2004.

Dorman L.I., "Cosmic Ray Interactions, Propagation, and Acceleration in Space Plasmas", Springer, 2006.

Dorman L.I., "Natural hazards for the Earth's civilization from space", Adv. in Geosciences, Volume 14, 281-286, 2008.

Dorman L.I., Cosmic Rays in Magnetospheres of the Earth and other Planets, Springer, Heidelberg, Germany, 2009.

Dorman L.I., Solar Neutrons and Related Phenomena, Springer, Heidelberg, Germany, 2010.

Dorman L.I., L.A. Pustil'nik, A. Sternlieb, and I.G. Zukerman, 2006a. "Forecasting of radiation hazard: 1. Alerts on great FEP events beginning; probabilities of false and missed alerts; on-line determination of solar energetic particle spectrum by using spectrographic method", Advances in Space Research, 37, 1124-1133.

Dorman L.I., N. Iucci, M. Murat, et al., 2006b "Forecasting of radiation hazard: 2. On-line determination of diffusion coefficient in the interplanetary space, time of ejection and energy spectrum at the source; on-line using of neutron monitor and satellite data", Advances in Space Research, 37, 1134-1140).

Gvozdevsky B. et al., "Longitude changes of the vertical geomagnetic cut off rigidities of cosmic rays, ICRC-2017.

Mirohnichenko L., 2015, "Solar Cosmic Ray. Fundamentals and Applications", Springer.

Parker E.N., 1965. Dynamical Processes in Interplanetary Space, Moscow, Inostrannaja Literatura (translation from English by L.I. Miroshnichenko, editor L.I. Dorman). 